\title{
Reframing the debate: a question of probability, not of likelihood ratio
}

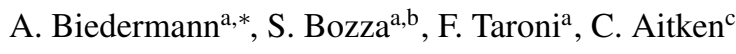

${ }^{a}$ University of Lausanne, School of Criminal Justice, 1015 Lausanne-Dorigny, Switzerland

${ }^{b}$ Ca'Foscari University Venice, Department of Economics, 30121 Venice, Italy

${ }^{c}$ University of Edinburgh, School of Mathematics and Maxwell Institute, Edinburgh, EH9 3FD, Scotland

\section{Abstract}

Evidential value is measured by a likelihood ratio. This ratio has two components, the probability, or probability 8 density, of the evidence if the prosecution proposition is true and the probability (density) of the evidence if the defence proposition is true. It takes the form of a single value, even if these probabilities are subjective measures of belief of the reporting forensic scientist.

Keywords: Uncertainty, Probability, Likelihood ratio, Evidence evaluation

\section{Introduction}

The title of this special issue of Science \& Justice raises the topic of precision in relation to likelihood ratios. The precision of the likelihood ratio is discussed in the context of its two components, the probability of the evidence if the prosecution proposition were true and the probability of the evidence if the defence proposition were true, with particular reference to probability as a subjective measure of belief. This interpretation of probability [8, 16] provides clear answers to questions about the nature and the properties of probability assertions. Most importantly, this interpretation is not imprecise, nor does it have the difficulties of interpretation that are associated with other forms of inference such as ones based on long-term repetitions of experiments under identical conditions. We also note that the laws of probability are precise and should not be confused with other measures of uncertainty that lack an ability to express uncertainty numerically. As a further starting point we emphasise that there is no way of validating an expert's single probability assessment [17], beyond agreement with the rules of probability.

This paper is structured as follows. In Sections 2 and 3, we emphasize that both likelihood ratios and probability assignments relate to the scientists' personal viewpoints. Section 4 clarifies the conceptual consequence of an inability to determine a single numerical value for a probability, an inability that can arise from a procedure known as incomplete probability elicitation. This section also recalls the distinction between the normative and descriptive view on probability, with an emphasis on the former, whereas Section 5 discusses the difference between precision and resolution. Sections 6 and 7 emphasise, respectively, the completeness of probability as a description of a given state of knowledge, at a given time, and the natural process of updating one's probability upon receipt of new data. Conclusions are presented in Section 8.

\section{Which or whose likelihood ratio?}

Advocates of the process in which there is imprecision in the measure of uncertainty argue that there are forensic systems that output likelihood ratios with differing values. It is argued that, as there is variation in the training data
*Corresponding author
Email address: alex.biedermann@unil.ch (A. Biedermann)

Preprint submitted to Elsevier 
from which the relevant probabilistic models are derived, this should be represented in the value, or values, reported 2 in court. This may well be the case in areas involving highly technical traces (e.g., audio recordings), involving subtle measuring instruments and complex procedures for the derivation of probabilities under competing propositions.

4 However, it is not the 'systems' that provide the report at trial, it is the scientist that provides the report. But then, what is the scientist to report at trial? There are three questions to be answered [e.g., 1, 10]. The first is: 'What is 6 the forensic scientist's probability for the forensic result (or, measurement, observation, etc.) if the first proposition, usually the one presented by the prosecution, is true and given the relevant conditioning information?' ${ }^{\text {. The second }}$

8 is: 'What is the forensic scientist's probability for the same forensic result if the second proposition, usually the one presented by the defence, is true and given the relevant conditioning information?'. The third question is: 'Is

10 the forensic result more probable, if the first proposition is true or if the second proposition is true?'. This triad of questions illustrates that, in order for the inference to be balanced, we must look at the forensic results from two 12 distinct perspectives. Each of these perspectives reduces to an expression of probability. Their combination in terms of a ratio forms the likelihood ratio. However, the combination is not essential to the current discussion. The focus

14 of attention in the first place, for forensic scientists, is the probability of the results, findings or evidence, given the proposition. This has been stated to be the most important lesson for the logic of evaluative forensic science [11].

${ }_{16}$ But what are those probabilities and how are they to be interpreted? These questions are of crucial importance to all expert forensic scientists and other participants in the judicial process. They require answers that are both practical and defensible.

\section{Not 'the' probability, but the scientist's probability}

In the absence of data, many scientists struggle with probability assignment and retreat to statements such as 'I do not know what the probability is'. Such a statement illustrates a lack of understanding of probability because probability is not something that can be known or not known. ${ }^{2}$ Instead, probability is a measure of belief in the occurrence of an event, and hence a measure of uncertainty because knowledge about the event is (nearly always) 24 incomplete. But with respect to whose knowledge is the uncertainty to be measured? It is with respect to the person who asserts the probability; in the current discussion that is the forensic scientist. Thus, it is the responsibility of the forensic scientist to express their probability, as the probability (measure of belief) does not exist per se as an external concept that can be investigated and known independently of an individual thinking mind. We disregard any position

28 that has arisen from the failure of a scientist to take personal responsibility for their probability assertions and their deference instead to some impersonal probability that is accepted by a scientific community as an 'objective' ${ }^{3}$ fact. Our interpretation of probability as a measure of belief is explicitly subjectivist [e.g., 19]. This choice of interpretation is not one of convenience. There are other interpretations of probability (e.g., those that consider various ideas of long32 run repetitions or relative frequencies), but we can safely leave them aside here because they all run into inferential difficulties [13]. The interpretation of probability as a measure of belief that is explicitly subjectivist is advocated here

34 because it satisfies an operational definition $[8,16]$, which is one that allows us to say what we mean: probability is your expression of your uncertainty about something, the truth or otherwise of a proposition, that is unknown to you. We note that this is the kind of question specified in the question triad mentioned in the previous section. There are two important features of this account of probability. The first is a theoretical feature, namely that personal probability assertions take the form of a single number. The second is a practical feature, namely that it can be shown that personal probabilities can feasibly be elicited, or even measured, by extremely simple and irrefutably coherent 40 schemes. ${ }^{4}$ Among these schemes are, for example, the perhaps somewhat arcane but compellingly efficient urn models [19].

\footnotetext{
${ }^{1}$ These questions must be reframed in terms of probability density functions whenever continuous measurements are available, otherwise each answer would have a point null probability.

${ }^{2}$ See [8] for a detailed discussion and rebuttal of assertions about non-knowledge of probabilities, and [4, 23] for further references on the same topic and reviews from a forensic science point of view.

${ }^{3}$ Our general position is that "(...) objectivity has no meaning in this context apart from that pragmatically endowed by thinking of it as a shorthand for subjective consensus" [3, p. 424], as noted also by Lindley ("Objectivity is merely subjectivity when nearly everyone agrees." [18, p. 87]).

${ }^{4}$ It is not necessary for the scope of this commentary to restate the derivation of these results which can be found in the original literature. For a discussion of the relevance of these results in forensic science contexts, in particular the discussion in this Special Issue, see our discussions in [24, 25].
} 
This interpretation of probability as a measure of belief also supports the representation of probability (measure 2 of uncertainty) as a single number. Thus there are several different independent routes that all lead to the same conclusion. These are fundamental results and it is important to remember that, whatever the source of a probability 4 assertion, or the way in which the assertion was achieved, it will always be possible to restate the reported probability in terms of the above probability measurement and elicitation devices (e.g., as a proportion of balls in an urn), thus 6 making explicit the personal position of the person issuing a probability statement. These are arguments that define those procedures as operational. ${ }^{5}$ It is not suggested, though, that forensic scientists should make urn models the 8 centre of their attention. Such models are conceptual devices that allow us to explain, and render explicit, what it may mean for a given person to say the assigned probability takes a particular single value.

In some cases, the forensic results may be seen as the outcome of a process about which the scientist has enough knowledge to formulate a mathematical model of a well-known form, such as a parametric probability distribution (e.g., in the context of certain types of transferable traces), or combinations of several distributions (e.g., in the case of glass fragments [7]). Once the parameter(s) of such models is (are) elicited, the required probability (density) 14 is obtained from the model. It will be a matter of judgement by the expert as to whether the probabilistic model is adequate, or what values for the model parameters ought to be chosen. Again, such a model has no meaning ${ }_{16}$ without reference to a particular person for whom the model is merely part of the process of formulation of an explicit probabilistic opinion about the value of the (forensic) outcomes and of a statement of that opinion in precise mathematical language. The model thus is a coherent and well-defined way in which one's probabilities may be distributed over the range of possible outcomes. This approach may also be considered to be the one which the scientist 20 considers appropriate for use as a function that maps the observed value of the forensic analysis to a probability, or probability density for continuous measurements.

\section{Incomplete probability elicitation: being clear what it means and what it does not mean}

The above comments are disputed by those scientists who contend that they cannot state their probability or, for whatever reason, refrain from assuming the responsibility of assigning probabilities in a given case. The difficulty experienced in practice of expressing uncertainty in terms of a single probability value is sometimes turned into a

26 critique of the concept, but this is unsound: any difficulty in comprehension of the concept does not make the concept wrong or deficient in any way. We must not confuse the challenge posed by use of the concept with deficiencies in its use [19]. Some scientists try to overcome the difficulty with the statement of a lower and upper value for their probability, rather than just a single value. But what would be the meaning of such a statement? Let us recall first that, by definition, different numbers or, in terms of the probability elicitation schemes mentioned in the previous section, proportions, represent distinct states of uncertainty. So, if a scientist does not assert a single probability, but 32 two numbers, then this is only a partial assertion of probability [16]. Scientists are, of course, free to limit their consideration to an incomplete probability assignment, but it is important then to recognise that this is not - as per the 4 definition of probability - equivalent to, and hence no substitute for, a genuine assignment of probability. Whether recipients of expert information should allow scientists to express themselves in terms of partial probability assertions 36 is not a matter for the theory of probability itself. The theory, of course, says nothing about how it is to be used. We just note that the question triad asks scientists to express their probabilities in a comparative way, leading to a single 38 likelihood ratio. If scientists cannot or are unwilling to express their probabilistic opinion, then this means - strictly speaking - that they are not able to provide any input for the evaluative stage. We also note that partial probability 40 assignments, such as upper and lower probabilities, provide no guidance to recipients of expert information as to how such pairs of values ought to be used, and even less so when inserted in both the numerator and the denominator of

42 a likelihood ratio. As a consequence, we cannot but conclude that adherence to an incomplete probability elicitation means it is not possible to evaluate the probative strength of forensic science results. It is for the judiciary to decide if

44 there is any merit in hearing such a report. Moreover, if the recipients of expert information have a difficulty with the presentation of probability as a single value, they will be even more perplexed by the question of how the scientist can justify the choice and reporting of two distinct values, and the interval that these two values imply. It may be thought sufficient to represent the value of the evidence as the one of the lower and upper values that is more supportive to

\footnotetext{
${ }^{5}$ The concept of operational definitions has origins in physics [e.g., 6], but see also [16] for further discussion.
} 
the defence and hence only one number. However, such an approach can be criticised by the choice of the width of 2 the interval, in a manner analogous to the width of a confidence interval (e.g., 95\%) in classical statistical inference. Hence, intervals are not a solution, they only aggravate what is a perceived problem in the first instance. There is no

4 compelling reason why a recipient of expert information might wish to receive anything else than the single value of the likelihood ratio, a value that is the best value that is obtainable with the knowledge available to the scientist. A 6 common criticism of the use of a single value is to say that there is uncertainty about the probabilities in the likelihood ratio and that to report a single value is to hide the existence of such uncertainty. However, it is important to reiterate 8 the fundamental understanding that it is not a reported probability that is uncertain. It is probability that is your measure of uncertainty about a quantity of interest (see also Section 6).

As an aside, note also that a benefit of a single value for the likelihood ratio as a measure of support for one proposition versus another proposition is the clarity with which the joint support for several pieces of evidence may 2 be evaluated. The posterior odds for one piece of evidence becomes the prior odds for the next piece of evidence, with due attention paid to conditionality. The combination of supports for different pieces of evidence is multiplicative. If 14 logarithms are used, the combination is additive with a pleasing analogy with a pair of scales for the weighing of evidence. It is difficult to envisage how interval supports for different pieces of evidence can be combined meaningfully.

The points raised in the above paragraphs relate to a confusion between descriptive and normative perspectives. That is, proponents who are unhappy over the difficulty of finding their single probability value as their expression of uncertainty seek to conceive of an additional technical means to describe the extent to which they are assured about the elicited number. ${ }^{6}$ Stated otherwise, they might say that the theory (of probability) does not well describe how they

20 intuitively feel about the problem. But probability does not claim to provide a descriptive account of people's unaided intuitions. Similarly, we will not invent new rules of addition to account, in a descriptive sense, for the intuitive 22 feeling of some people that $2+2$ could give anything else than the normative result of 4 . The justification for the use of values that are numerically precise is that of a normative point of view, that is, to make explicit a coherent thought process about real-world events. Confusion over the normative and descriptive approaches are the source of many misconceptions about probability and erroneous claims against the use of probability for forensic science applications 26 [5] and in other fields [e.g., 2, 20, 21].

\section{A question of resolution, not precision}

In the evaluation of evidence there is no concept of a long-term repetition of an experiment for the measurement of a characteristic from which an estimate of the natural variation, and hence precision, of the measurement may so be obtained. There may be discussion about whether it is feasible and desirable to determine if a single probability should be given to, for example, four significant figures and hence make a distinction between 0.0101 and 0.0102 .

32 This is a question of the level of resolution, not of precision. The probabilities 0.0101 and 0.0102 are different and hence, by definition, express different levels of uncertainty. However, on practical grounds, evidential value should be 4 considered as measurable in increments which are no smaller than that which may be thought discernible by a juror or other fact-finder. This connects to the historical notion of ban, used to name units of information, with deciban

36 being considered as the smallest change in evidential value that is perceptible to humans [12]. Consider a probability value of 1 as a numerator for a likelihood ratio. For denominators of $0.01,0.0101$ and 0.0102 , the evidential values 38 are 100, 99.0099 and 98.03922, respectively. It is questionable whether a human being could separate meaningfully these three different evidential values.

\section{Your probability is about your uncertainty, but you are not uncertain about your probability}

When a scientist assigns their probability for a proposition about which they are uncertain, it is not necessary to 42 assign a precision to the probability thus provided. A probability has been assigned as a measure of uncertainty in the belief of a proposition. There is no need to assign a measure of uncertainty to the measure of uncertainty.

At times, assigning one's probability may be hard in the sense that one may hesitate to accept or stick to a particular value. The reasons for this may be diverse. A recurrent example is differences in the contents of the knowledge base

${ }^{6}$ For research on how to represent personal degrees of beliefs using intervals rather than single numerical assignments, see for example [9, 22]. 
upon which a probability assignment is based. However, this does not justify a statement of the kind 'I am uncertain 2 about my probability'. Such a statement is unjustified because your probability is your complete expression of your uncertainty and you are not going to place a probability on your probability. This would only lead to an endless

4 regress [19] which, in essence, is but an illusionary dismissal of uncertainty [24]. ${ }^{7}$ Of course, the same probability assignment may be based on different amounts of data (e.g., a limited or an extensive database), but this does not 6 make the probability assignment in one case 'more probable' than in the other, or 'more likely to be close to the right probability'. This is so, essentially because there is no 'right' or 'true' probability.

In either case, small or large amounts of data and other knowledge, the particular probability assignment determined is the best representation of one's state of uncertainty, and this is why it is brought to the attention of the recipient of expert information. We see no convincing reason why one should report anything else (which includes partial probability assertions) than the best representation of one's beliefs. This does not prevent scientists from men2 tioning that their beliefs may be based on limited amounts of data. The features of the knowledge base relied upon is something that has still merit to be disclosed. Generally, it is desirable that reported probabilities be accompanied with

14 information to help recipients of expert information understand how and on what bases scientists have reached their conclusions. Hence, contrary to what some critics assert, a single probability assignment and a report of such does

16 not amount to the with-holding of information or to the conveyance of false impressions of precision. As explained above, evaluation of evidence is about resolution not precision.

\section{7. Analysing reality, not merely describing it}

'What is my probability for an unknown person (or object) drawn from this population to have the analytical 20 feature $F$ ?' Such a question ${ }^{8}$ is commonly answered on the basis of the proportion $\theta$ of the population that has feature $F$, that is $\operatorname{Pr}(F \mid \theta)$. To learn about the population proportion $\theta$, scientists may investigate measurements $22 \mathbf{x}=\left\{x_{1}, \ldots, x_{m}\right\}$ on a sample of $m$ individuals from this population of interest, and then obtain a (probability) distribution over the possible values for the parameter of interest $\theta$ (i.e., a probability density function $f(\theta \mid \mathbf{x})$ ). According to this argument, personal beliefs concerning the analytical feature for an unknown person drawn from the relevant population can be computed as $\operatorname{Pr}(F \mid \mathbf{x})=\int \operatorname{Pr}(F \mid \theta) f(\theta \mid \mathbf{x}) d \theta$.

Consider now another sample of $n$ individuals taken from the same population of interest with no overlap with the previous sample. Denote the measurements from this other sample as $\mathbf{y}=\left\{y_{1}, \ldots, y_{n}\right\}$. The resulting posterior 28 distribution $f(\theta \mid \mathbf{y})$ of $\theta$ will almost certainly be different; it may be of the same form but with posterior parameters that will be different from those obtained from the first sample. Thus, the probability of interest, i.e. $\operatorname{Pr}(F \mid \mathbf{y})=$ 30 $\int \operatorname{Pr}(F \mid \theta) f(\theta \mid \mathbf{y}) d \theta$ will also be different.

While this is no source of concern in statistical science, because it rather shows that the approach is indeed sensible 32 to variation in the input information, this appears to trouble some quarters in forensic science. A widely raised point starts with an observation of the kind 'if we take another sample from the same population, the assignment would have 34 been different' and that concludes by asserting 'hence there is a problem, variability in the procedure, and we do not know what the right (or, correct) value is'. While we agree with the former statement, which is merely a description 36 of reality, we disagree with the latter. It is all but natural that different data may shape one's belief differently, but we do not have a collection of disconnected beliefs about the same event (or, parameter) based on distinct sets of data.

${ }_{38}$ We have a distribution about the population parameter that we continuously update, in a sequential way. We start with one dataset (e.g., $\mathbf{x}$ ), which will reshape our initial belief in one way or another, and then use this as a starting 40 point before considering a further data set (e.g., y), which may add to the direction taken based on the previously seen data, or lead us in another direction. This means analysing reality, that is revising our belief state about the real world

42 based on new data, rather than merely describing it, such as in terms of distinct subsets of the population that may lead to variable findings. These observations are all standard elements of Bayesian theory. In this Bayesian account of 44 information processing, one's current belief state provides a complete account of the information at one's disposal at a given time. The fact that there may be other information available, or that consideration of further information may

\footnotetext{
${ }^{7}$ Similarly, as noted in Section 4, it appears illusionary to claim an interval, that is two values, as a substitute for the difficulty of specifying a single value.

${ }^{8}$ Answering such a question may be necessary, for example, in order to assign a value for the denominator of the likelihood ratio.
} 
lead to another belief state, is of no detriment to the procedure and one's current state of mind. Any other argument 2 bears a risk of running into frequentist ideas that consider possible values that are not actually observed. It is crucial to understand that if further data become available, then we have a new situation and we are prepared and able to

4 update our belief state. This will not mean that one's previous belief state was invalid or wrong, it solely means that it requires updating in the light of the newly acquired information. In the example described above with two samples,

$6 \quad \mathbf{x}$ and $\mathbf{y}$, they can be combined to give a revised evidential value based on a sample of size $m+n$.

The probabilities described in the previous paragraphs integrated out the uncertainty in $\theta$. Thus, the probability

8 obtained does not include explicit information about $\theta$. However, information about the distribution of $\theta$ is of no interest in itself. The information available for the determination of the measure of belief in $F$ dependent on $\mathbf{x}$ 10 or $\mathbf{y}$ is available in $\operatorname{Pr}(F \mid \mathbf{x})$ and $\operatorname{Pr}(F \mid \mathbf{y})$. As we have reiterated in [25], there is no information that has been concealed or lost. The probability of interest which has been computed with an integration over the uncertain 12 parameter, incorporates all the available knowledge at a given instant of time. Moreover, one must not confuse situations in which the object of reporting is the probability of the scientific result, such as the observation $F$, and 14 situations in which the object of reporting is a parameter $\theta$ (e.g., the proportion of a seizure that contains something illegal).

\section{Conclusions: What should forensic scientists do (next)?}

The expression of the probative strength of forensic results in terms of likelihood ratios has never been discussed as widely in forensic science in the past as now. The development has perhaps taken place too rapidly and some fundamental topics need further examination. Indeed, experience demonstrates that while many contributors have difficulty in explaining that a likelihood ratio involves two conditional probabilities, the question of what is understood by probability often turns out to be surprisingly perplexing. Yet, if we wish to discuss likelihood ratios, it 22 appears natural in the first place to require a clear view about the ingredients of likelihood ratios, that is probabilities. A clear definition of probability is a fundamental issue yet it appears there is no agreement in forensic science as to what this may be [4]. The previous sections discuss recurrent aspects of this topic but there is nothing new in terms of the fundamental principles we have used, as in our previous discussion in [24] about the same topic. The existing literature. Other accounts of probability are unable to account for all uncertainty as encountered in practice, and hence cannot be used in certain situations. There are also inferential difficulties and 8 among these is consideration of problems of precision.

A new challenge for forensic science is the development of a fundamental understanding of probability and the measure of uncertainty. Not only is it difficult to understand probability in the first place, though excellent accounts are available on this [e.g., 19], it is at least as difficult to make meaningful use of that fundamental understanding once 2 it has been acquired. The difficulty of the former task, the comprehension of probability, even by expert statisticians, is well illustrated by the following comment by Lindley when asked what, in his opinion, was the most important book on statistics:

"de Finetti's two-volume work. I have seriously suggested that all statisticians should give up research for two years and read one volume a year. It is a difficult book but properly understood it could change your life." [14, at p. 75]

It is necessary that forensic scientists have statisticians at their disposal to help them understand uncertainty and use probability appropriately. Such understanding and use are vital preliminaries for a proper approach to the use likelihood ratio for the evaluation and interpretation of evidence. Forensic scientists, with their initial deep understanding of the evaluative problem and the nature of the results of forensic examinations, need to engage in an open-minded exchange with statisticians, mathematicians and philosophers of science who are proficient in the mastery of formal methods of reasoning. Such an interdisciplinary approach is necessary to ensure conflicts in the concepts of forensic interpretation are critically examined and resolved.

\footnotetext{
${ }^{9}$ Note that this is a simplification made for the sole purpose of ease of presentation. Likelihood ratios may involve multiple propositions and combinations of different probabilities [15].
} 


\section{Acknowledgements}

The authors acknowledge the support of the Swiss National Science Foundation through grant No. BSSGI0_155809 and the University of Lausanne.

4 [1] C G G Aitken, $\mathrm{P}$ Roberts, and G Jackson. Fundamentals of Probability and Statistical Evidence in Criminal Proceedings (Practitioner Guide No. 1), Guidance for Judges, Lawyers, Forensic Scientists and Expert Witnesses, Royal Statistical Society's Working Group on Statistics and the Law, 2010. www.rss.org.uk/Images/PDF/influencing-change/rss-fundamentals-probability-statistical-evidence.pdf

[2] J Baron. Thinking and Deciding. Cambridge University Press, New York, 4th edition, 2008.

[3] J M Bernardo and A F M Smith. Bayesian Theory. John Wiley \& Sons, Chichester, second edition, 2000.

14] A Biedermann. The role of the subjectivist position in the probabilization of forensic science. Journal of Forensic Science and Medicine, 1: $140-148,2015$

12 [5] A Biedermann, F Taroni, and C Aitken. Liberties and constraints of the normative approach to evaluation and decision in forensic science: a discussion towards overcoming some common misconceptions. Law, Probability and Risk, 13:181-191, 2014.

14 [6] P Bridgman. The Logic of Modern Physics. Macmillan, New York, 1927.

[7] J M Curran, C M Triggs, J Buckleton, K A J Walsh, and T Hicks. Assessing transfer probabilities in a Bayesian interpretation of forensic glass evidence. Science \& Justice, 38:15-21, 1998.

[8] B de Finetti. Theory of Probability, A Critical Introductory Treatment, Volume 1. John Wiley \& Sons, London, 1974

18 [9] A P Dempster. Upper and lower probabilities induced by a multivalued mapping. Annals of Mathematical Statistics, 8:325-339, 1967.

[10] ENFSI. ENFSI guideline for evaluative reporting in forensic science, Strengthening the evaluation of forensic results across Europe (STEOFRAE). Dublin, 2015

[11] I W Evett. Evaluation and professionalism. Science \& Justice, 49:159-160, 2009.

22 [12] A Hodges. Alan Turing: Enigma. Vintage Books, London, first edition, 1992

[13] C Howson and P Urbach. Scientific Reasoning: The Bayesian Approach. Open Court, 3rd edition, 2005.

24 [14] H Joyce. Bayesian thoughts. Communication with Dennis Lindley. significance, 1:73-75, June 2004.

[15] R E Kass and A E Raftery. Bayes factors. Journal of the American Statistical Association, 90:773-795, 1995.

26 [16] F Lad. Operational Subjective Statistical Methods : a Mathematical, Philosophical, and Historical Introduction. John Wiley \& Sons, New York, 1996.

17] D V Lindley. On the reconciliation of probability assessments. Journal of the Royal Statistical Society. Series A (General), 142:146-180, 1979.

30 [18] D V Lindley. That wretched prior. Significance, 1:85-87, June 2004.

[19] D V Lindley. Understanding Uncertainty. John Wiley \& Sons, Hoboken, revised edition, 2014.

32 [20] M Oaksford. Normativity, interpretation, and Bayesian models. Frontiers in Psychology, 5:1-5, 2014.

[21] M Oaksford and N Chater. Bayesian rationality: the probabilistic approach to human reasoning. Oxford University Press, Oxford, 2007.

34 [22] P Suppes and M Zanotti. Conditions on upper and lower probabilities to imply probabilities. Erkenntnis, 31:163-169, 1989.

[23] F Taroni, C G G Aitken, and P Garbolino. De Finetti's subjectivism, the assessment of probabilities and the evaluation of evidence: A commentary for forensic scientists. Science \& Justice, 41:145-150, 2001

[24] F Taroni, S Bozza, A Biedermann, and C Aitken. Dismissal of the illusion of uncertainty in the assessment of a likelihood ratio. Law, Probability and Risk, 15:1-16, 2016.

[25] F Taroni, S Bozza, A Biedermann, and C Aitken. Rejoinder. Law, Probability and Risk, 15:31-34, 2016. 